\title{
Evolución de la segregación residencial y condiciones de habitabilidad de las viviendas de los adultos mayores en la ciudad de Córdoba, Argentina \\ ENRIQUE PELÁEZ
}

> CIECS, CONICET y UNC, Córdoba, Argentina. enpelaez@gmail.com

FLORENCIA MOLINATTI

> CIECS, CONICET y UNC, Córdoba, Argentina. fmolinatti@hotmail.com

Universidad de Valparaíso

Facultad de Arquitectura

Revista Márgenes

Espacio Arte Sociedad

Evolución de la segregación residencial y condiciones de habitabilidad

de las viviendas de los adultos mayores en la ciudad de Córdoba, Argentina

Diciembre 2016 Vol $13 \mathrm{~N}^{\circ} 19$

Páginas 7 a 20

ISSN elec. 0719-4463

ISSN imp. 0718-4034

Recepción: agosto 2016

Aceptación: noviembre 2016

\section{RESUMEN}

Las ciudades latinoamericanas experimentan un proceso acelerado y sin precedentes de envejecimiento de su población, lo cual implicará importantes desafíos para la sociedad y sus gobiernos. La dimensión demográfica de este proceso ha sido ampliamente documentada en la literatura especializada, pero poco se conoce sobre su dimensión urbana. Este trabajo tiene por objetivo analizar, utilizando datos censales, los niveles y patrones de la segregación residencial en la población adulta mayor al interior de ciudad de Córdoba en 1991, 2001 y 2010, y analizar las condiciones de habitabilidad de las viviendas de los adultos mayores en los conglomerados envejecidos.

Los resultados indican que los adultos mayores se encuentran altamente segregados en la ciudad de Córdoba. Este grupo poblacional ocupa zonas específicas en el espacio urbano, entre las cuales se evidencian grandes heterogeneidades como consecuencia de los procesos urbanos y demográficos que han moldeado y cambiado los perfiles de los espacios residenciales de la ciudad. Hacia 2010 se observa un menor número de espacios residenciales con máximas de concentración de adultos mayores en el área central y en algunos barrios pericentrales con un marcado proceso de gentrificación y una mayor presencia de conglomerados residenciales en los barrios tradicionales localizados en áreas intermedias y en el corredor noroeste, caracterizados por un proceso de despoblamiento.

PALABRAS CLAVE

envejecimiento demográfico, segregación por edad, vivienda adecuada, conglomerados residenciales

Evolution of residential segregation and living conditions in senior's residences in the city of Córdoba, Argentina

ABSTRACT

Latin American cities are experiencing an accelerated and unprecedented process of population aging, which implies important challenges for their societies and governments. The demographical magnitude of this process has been thoroughly documented in specialized literature, but there's little research regarding its urban dimension. This work's objective is to analyze, through censal data, the levels and patters of residential segregation in senior populations in the city of Córdoba during 1991, 2001 and 2010, and to analyze the living conditions of senior living spaces in aged conglomerates.

Results indicate that seniors are disproportionally segregated in the city of Córdoba. This population occupies specific zones in the urban space, among which extensive heterogeneity is evident as a consequence of urban and demographic processes which have molded and changed the residential space profile in the city. In 2010 fewer residential spaces with high concentration of seniors can be found in the central and pericentral neighborhoods with an increased gentrification process, and a greater concentration can be found in traditional neighborhoods in intermediate areas and on the northeastern corridor, both in the middle of a depopulation process. KEYWORDS

demographical aging, age segregation, fitting housing, residential conglomerates

Evolución de la segregación residencial y condiciones de habitabilidad de las viviendas de los adultos mayores en la ciudad de Córdoba, Argentina > Enrique Peláez, Florencia Molinatti
Revista Márgenes № 19 Vol 13 > Diciembre $2016>7$ Facultad de Arquitectura > Universidad de Valparaíso 


\section{INTRODUCCIÓN}

Pese a las diferencias entre países, el proceso de envejecimiento de la población constituye un proceso generalizado y sin precedentes en todo el mundo, y en particular en América Latina y el Caribe, en donde la velocidad del proceso supera al resto de las regiones del planeta, con importantes implicancias para los gobiernos y la sociedad. De acuerdo a la Comisión Económica para América Latina y el Caribe (CEPAL):

En América Latina el número de adultos mayores superará por primera vez al de niños en 2040. Se pasa así de una estructura de población joven en 1950 a una población en claro proceso de envejecimiento; en 2010 ya se observa claramente la disminución del peso relativo de la población menor de 15 años en el total de la población, de un 40\% en 1950 a un $28 \%$ en 2010 . Se espera que represente solo un 15\% de la población total en 2100 (CEPAL, 2011:14).

Si bien el envejecimiento se produce en la región a un ritmo más acelerado de lo históricamente observado en los países más desarrollados, lo más preocupante es que ocurre:

En un contexto caracterizado por una alta incidencia de pobreza, una persistente y aguda inequidad social, un escaso desarrollo institucional, una baja cobertura de la seguridad social y una probable tendencia hacia la disminución del tamaño de las fuentes de apoyo producto de los cambios en la estructura y composición familiar (CEPAL, 2004:1).

Este acelerado proceso de envejecimiento demográfico implicará retos notables para la sociedad en materia de cuidado de los adultos mayores, particularmente en lo referido a la dimensión urbana. Las ciudades, que concentrarán de manera creciente la población del país, incluyendo a la envejecida, fueron originalmente pensadas y diseñadas para sujetos con capacidades plenas y hoy enfrentan a un nuevo tipo de habitante, la población adulta mayor. Se requieren ajustes en el diseño de la ciudad a los requerimientos de este nuevo tipo de usuario. Hoy en día se manifiestan una serie de fallas de calidad urbana que afectan el bienestar de la población adulta mayor: localización y diseño de los grandes supermercados que requieren grandes esfuerzos físicos para llegar y luego utilizar el servicio; sistema de transporte público masivo (por ejemplo, paradas inadecuadas, escalones altos para subir y bajar del ómnibus, movimientos bruscos); tiempos de los semáforos; ausencia de espacios peatonales y estrechas aceras; entre otras (Garrocho y Campos, 2005).

Asimismo, la vejez significa un deterioro y pérdida no sólo de las capacidades físicas, sino también de las financieras, que acentúan su vulnerabilidad, dependencia, discriminación y aislamiento. A medida que aumenta la esperanza de vida, también lo hacen las probabilidades que diferentes generaciones de una familia estén vivas al mismo tiempo. Sin embargo, estas generaciones tienen mayores probabilidades de vivir por separado. Las personas mayores que viven solas tienen mayores riesgos de aislamiento y suicidio, como así también de pobreza (Organización Mundial de la Salud - OMS, 2015). Un estudio comparativo para América Latina y el Caribe señala la desventaja relativa de los adultos mayores que viven solos en términos de bienestar material, particularmente en los países con niveles económicos menos avanzados, siendo la corresidencia una estrategia de apoyo a los ancianos con mayores niveles de vulnerabilidad (Saad, 2005). Sin embargo, consideran- do la gran heterogeneidad entre los países de la región esto podría ser relativo, ya que los adultos mayores que se encuentran en buen estado de salud y son independientes para las actividades de la vida diaria podrían optar por vivir solos o en pareja, especialmente en los países más avanzados en la transición demográfica, tales como Argentina (Peláez y Féliz-Ferreras, 2010).

Los adultos mayores, junto con los niños, migrantes y otros grupos vulnerables, conforman un segmento de población con serias dificultades para acceder a una vivienda adecuada y asequible. Garantizar que las personas mayores vivan en una vivienda adecuada se asocia con un mejor estado de salud y posiblemente, promueva mejores relaciones sociales dentro y fuera del hogar (OMS, 2015). En algunos países latinoamericanos, como Brasil, Ecuador y México, aún se observan serios rezagos en el acceso a servicios básicos (agua por red y drenaje) y las condiciones materiales y de equipamiento de la vivienda no son las mejores (Garay Villegas et al., 2015). De acuerdo a las autoras, esto representa un reto importante para el análisis de los entornos físicos y sociales, pues no se puede avanzar en este sentido si las necesidades más básicas no están cubiertas (Garay Villegas et al., 2015:123).

Sin embargo, apenas se ha explorado sobre la dimensión urbana del envejecimiento y poco se sabe de la relación funcional entre la ciudad y la población adulta mayor. Es por ello que parece pertinente abordar la relación entre envejecimiento poblacional y ciudad, tomando la problemática de la segregación residencial como eje de análisis. El objetivo de este trabajo es analizar los niveles y patrones de la segregación residencial en la población adulta mayor al interior de ciudad de Córdoba ${ }^{1}$ en 1991, 2001 y 2010, y analizar las condiciones de habitabilidad de las viviendas de los adultos mayores en los conglomerados envejecidos.

\section{ASPECTOS CONCEPTUALES}

\section{Envejecimiento demográfico}

Uno de los efectos más significativos de las transformaciones que ha producido la dinámica demográfica de los países de América Latina y el Caribe es la reestructuración etaria de la población y el consiguiente envejecimiento de la población. Desde el punto de vista demográfico, el envejecimiento significa: por un lado, el aumento del peso relativo de las personas mayores de 60 años en la población total y, por otro, lado, el un aumento de la longevidad (CEPAL, 2004, 2011).

El estudio del proceso de envejecimiento plantea desafíos teóricos y metodológicos, ya que no existe un único paradigma de la vejez y el envejecimiento: la vejez alude a una realidad multifacética atravesada no sólo por el paso del calendario, sino también por aspectos fisiológicos, sociales y culturales (Centro Latinoamericano y Caribeño de Demografía - CELADE, 2006:14).

Es preciso establecer una distinción entre los aspectos cronológicos de la vejez y su construcción social. A pesar que cada sociedad establece el límite de edad a partir de la cual una persona es considerada mayor, la vejez está relaciona con la edad fisiológica y se correlaciona con la pérdida de ciertas capacidades instrumentales y funcionales para mantener la autonomía y la independencia. De acuerdo al CELADE:

En este contexto, la vejez puede ser tanto una etapa de pérdidas como de plenitud, todo depende de la combinación de recursos y la estructura de oportunidades individuales y 
generacionales a la que están expuestos los individuos en el transcurso de su vida, de acuerdo a su condición y posición al interior de la sociedad. Esto remite a una conjugación de la edad con otras diferencias sociales -tales como el género, la clase social o la etnicidad-que condicionan el acceso y disfrute de dichos recursos y oportunidades (2006:14).

En la literatura regional e internacional, la dimensión demográfica del envejecimiento ha sido amplia y profundamente estudiada, pero poco se conoce sobre las demás dimensiones del fenómeno, siendo la urbana una de las dimensiones menos estudiadas (Garay Villegas, Montes de Oca Zavala y Hebrero Martínez, 2015; Garrocho y Campos, 2005, CELADE, 2006).

Como consecuencia del aumento absoluto y relativo de la población mayor, y de su concentración en ciudades, han surgido nuevos enfoques para analizar las estructuras y procesos espaciales de la vejez: por un lado, el urbanismo gerontológico (Bosch Meda, 2006, 2013; Narváez Montoya, 2011), y por otro, lado, la geografía gerontológica (Andrews et al., 2007).

Particularmente, América Latina y el Caribe es una región con grandes avances desde el punto de vista de los acuerdos regionales sobre los derechos de los adultos mayores. Es la primera región en el mundo en tener una Convención específica sobre los derechos de los adultos mayores, la Convención Interamericana sobre la Protección de los Derechos Humanos de las Personas Mayores aprobada en 2015 en el marco de la Organización de los Estados Americanos (OEA) y hasta ahora ha sido ratificada por Argentina, Bolivia, Brasil, Chile, Costa Rica y Uruguay.

Sin embargo, a pesar de que en América Latina y en Argentina, particularmente, el envejecimiento poblacional será uno de los fenómenos urbanos más trascendentes del siglo XXI, poco se ha explorado sobre la segregación residencial de los adultos mayores que tiene consecuencias relevantes en términos del bienestar de la población mayor, de la cohesión social y de la planeación socioespacial de las ciudades.

\section{Segregación residencial}

Brun (1994), quien ha hecho numerosas contribuciones epistemológicas sobre el significado del concepto “segregación”, argumenta que esta noción no es lo suficientemente precisa como para ser utilizada como una herramienta conceptual rigurosa. La polisemia y la ambigüedad que caracteriza este concepto, debido a la fuerte orientación semántica que ha cargado la palabra desde sus orígenes, invitan a los investigadores y estudiosos del tema a contextualizar el uso de este término. En todos los casos, el sentido es dado, en gran parte, por el tipo de aproximación que se quiera hacer del tema. Desde luego, no es un concepto unívoco, el término tiene diversas dimensiones y utilizaciones (Brun, 1994).

En su sentido etimológico, la segregación, de acuerdo a la definición de la Real Academia Española, resulta de la acción de separar o de apartar algo de otra u otras cosas. Sin embargo, esta idea puede ser entendida de diversas formas. Desde el punto de vista sociológico, la palabra puede significar la ausencia de interacción entre grupos sociales; en cambio, cuando es avocada en un sentido geográfico, segregación puede referirse a la desigualdad en la distribución de los grupos sociales a través del espacio físico. La presencia de un tipo de segregación no implica necesariamente la presencia de la otra. Por ejemplo, en la sociedad de castas la segregación sociológica puede ser casi absoluta, aun cuando los miembros de las diferentes castas estén próximos en el espacio físico. No obstante, es probable que ambos tipos de segregación estén correlacionadas (White, 1983).

Grafmeyer (1994) considera que si se circunscribe a la segregación a su dimensión residencial pueden encontrarse tres abordajes interdependientes, pero conceptualmente distintos, de dicha noción:

- $\quad$ El primero relaciona directamente el tema de la segregación con la figura del ghetto, donde la separación física está institucionalizada y es preservada autoritariamente como principio fundador de la organización social.

- $\quad$ El segundo se refiere a la identificación de las diferencias de localización entre grupos definidos en función de criterios tales como la posición social, el origen étnico, la religión, la edad, etc. Un grupo de población se considera segregado cuando se encuentra distribuido de forma desigual entre las diferentes zonas de una ciudad. Este abordaje presenta una larga tradición, especialmente en la literatura de lengua inglesa.

- $\quad$ El tercero pone acento sobre las oportunidades desiguales de acceso a los bienes materiales y simbólicos ofrecidos por la ciudad. Los principales referentes de este abordaje se encuentran en el Centro de Sociología Urbana de Francia.

En términos generales, la noción de segregación, "sin apellido" (Rodríguez Vignoli, 2001), remite a la existencia de diferencias o desigualdades dentro de un colectivo social y a la separación de los sujetos en categorías que tienen cierto grado de distinción jerárquica o valorativa. La denominación "residencial" circunscribe el ámbito de referencia del concepto, ya que se refiere a las condiciones de localización cotidiana de las personas o sus familias que residen en un determinado aglomerado urbano. La segregación residencial tiene lugar cuando las disparidades o diferencias existentes en un colectivo tienen una expresión territorial o geográfica.

\section{Relación entre envejecimiento demográfico y segregación residencial}

La dimensión urbana del envejecimiento demográfico ha sido poco explorada y los escasos estudios sobre los niveles y procesos de segregación residencial en la población adulta mayor provienen mayormente de Asia y del Pacífico (Andrews et al., 2007) y de Ios Estados Unidos (Stanford Center on Longevity, 2015; Winkler y Klass, 2012). Algunos estudios evidencian la existencia de segregación por edad, particularmente entre la población adulta mayor y los adultos jóvenes. Generalmente, estos grupos residen en barrios distintos y con poca interacción entre sí (Winkler y Klass, 2012). Otros estudios realizados en los Estados Unidos y España dan cuenta de las influencias del vecindario sobre la satisfacción, el bienestar y la mortalidad de adultos mayores (Balfour y Kaplan, 2002; Bassuk, Berkman, \&Amick, 2002; Glass \& Balfour, 2003; Pérez et al., 2001, citados en Andrews et al., 2007).

De acuerdo a estos estudios, la desventaja de la segregación por edad es que obstaculiza las oportunidades básicas para que las personas se conozcan e interactúen. Según Hagestad y Uhlenberg (2006), la segregación por edad tiene cuatro importantes consecuencias que requieren atención: En primer lugar, produce y reproduce la discriminación por edad. En segundo lugar, es una amenaza a la integración y aumenta el riesgo de aislamiento. En tercer lugar, impide la socialización entre los jóvenes y viejos. En cuarto lugar, impide la generativity ${ }^{2}$ en especial la creatividad y el 
mantenimiento de una sociedad generadora (Hagestad y Uhlenberg, 2006:643, traducción propia).

Particularmente, para los adultos mayores la segregación por edad afecta las redes sociales y a su vez la disponibilidad de que la persona de edad mayor reciba apoyo social. Aunque los servicios dirigidos a grupos de edad en particular son necesarios y tienen su propósito, es importante crear espacios y programas intergeneracionales para promover la integración social y el intercambio de beneficios entre personas de diferentes edades (Hagestad y Uhlenberg, 2006). En América Latina y el Caribe, particularmente, el rol de la familia como apoyo de los adultos mayores ha sido fundamental en un contexto con grandes falencias en los servicios de protección social brindados por el Estado, especialmente en aquellos países con niveles económicos menos avanzados (Saad, 2005).

En América Latina los antecedentes son más recientes y se concentran en algunas pocas ciudades, tales como la Ciudad de México (Garrocho y Campos, 2015; Negrete Salas, 2003); el Área Metropolitana de Toluca, México (Garrocho y Campos, 2005); Bahía Blanca, Argentina (Formiga y Prieto, 2008; Prieto, 2010); y ciudad de Córdoba, Argentina (Peláez, 2005, 2006).

Particularmente en Argentina, un estudio realizado en Bahía Blanca evidencia un aumento de segregación espacial correspondiente a los adultos mayores de 65 años y más en dicha ciudad en el período inter-censal 1991 - 2001. La localización residencial de la población adulta mayor muestra una distribución homogénea sobre el territorio y se encuentran concentrados en el centro de la ciudad y en coincidencia con sectores tradicionales de la ciudad en relación a determinadas actividades que caracterizaron dichas áreas como la actividad férrea (Prieto, 2010).

Otro estudio realizado para este país, pero sobre la ciudad de Córdoba, ciudad objeto de estudio en el presente artículo, señala, también para el período 1991-2001, que en la evolución de la estructura etaria se observa un envejecimiento paulatino en la mayoría de los sectores de la ciudad, a excepción de algunos sectores periféricos, y el barrio central de Nueva Córdoba que por la gran concentración de estudiantes ha disminuido su edad mediana. Respecto a la relación la localización residencial y la vulnerabilidad del adulto mayor, el trabajo evidencia los clusters con nivel socioeconómico bajo concentran a la mayoría de la población pero no a la mayoría de los adultos mayores. Esto confirma la hipótesis de que los adultos mayores tienen una mejor situación socioeconómica que los jóvenes, como sobrevivientes de un estado de bienestar (Peláez, 2005, 2006).

Un tema muy vinculado a la problemática de la segregación residencial es el acceso a una vivienda adecuada y asequible por parte de la población adulta mayor. Un estudio comparado entre América Latina y España indica que:

Disfrutar de una vivienda adecuada, vivir en un ambiente seguro y saludable, y permanecer en el propio hogar durante el máximo tiempo posible, en función de las preferencias y necesidades personales, son básicamente los criterios considerados en relación con el derecho a la vivienda y el entorno saludable de dicho grupo de población (Redondo et al., 2015:224).

La Convención Interamericana sobre la Protección de los Derechos Humanos de las Personas Mayores, primer tratado internacional que acuerda una serie de derechos específicos para las personas mayores, ofrece una interpretación de los derechos humanos en el contexto del envejecimiento y estandariza derechos notablemente importantes que no han sido considerados de manera explícita por otro instrumento internacional. Entre otros derechos, habla del derecho a la vivienda, el derecho a un ambiente sano, el derecho a la accesibilidad y a la movilidad personal.

De acuerdo a Redondo y colaboradores, el envejecimiento demográfico y las políticas de protección social de la vejez son determinantes de los tipos de hogares y arreglos de convivencia que establecen las poblaciones de adultos mayores. En este sentido, las diferentes modalidades de allegamiento residencial de los adultos mayores constituyen una dimensión clave para estimar el tipo y la calidad del apoyo que reciben las personas que pueden requerir ayuda para su desempeño cotidiano (Redondo et al., 2015).

Tradicionalmente se ha entendido que la familia extensa, en que conviven por lo menos dos generaciones, es la que proporciona el sistema de cuidado informal más eficiente, ya que permite distribuir la carga de cuidados entre un mayor número de personas. Sin embargo, la evidencia empírica sobre la situación social en los países latinoamericanos cuestiona esta presunción, según la cual la familia extensa suele ser una forma de arreglo residencial ante la escasez de recursos que permite mantener hogares autónomos (Redondo et al., 2015). Según estos autores, el riesgo de desprotección, abandono o falta de cuidados aumenta en los hogares unipersonales de personas mayores, si no poseen recursos económicos suficientes para proveerse de servicios formales oportunos y de calidad (Redondo et al., 2015:239).

De acuerdo a Saad (2005), a diferencia de lo observado en los países más desarrollados, para la mayoría de los adultos mayores de América Latina y el Caribe vivir solo representaría más un riesgo asociado a la falta de una mejor opción. La corresidencia desempeña un rol fundamental en la garantización del soporte necesario de los adultos mayores más vulnerables. Un estudio realizado sobre los arreglos residenciales de los adultos mayores en Argentina y República Dominicana da cuenta que mientras en el primer país existe una mayor prevalencia de hogares nucleares y unipersonales, mientras que en el segundo priman los hogares extendidos (Peláez y Féliz-Ferreras (2010). De acuerdo a estos autores, las diferencias entre ambos países tienen dos explicaciones: por un lado, una tendencia más arraigada en República Dominicana a no dejar que los ancianos vivan solos, por lo que pasan a vivir en hogares extendidos, $y$, por otro lado en Argentina, una gran proporción de adultos mayores están en una posición financiera tal que les permite seguir viviendo en hogares nucleares sin importantes limitaciones socioeconómicas.

Los resultados de otro estudio sobre las condiciones de la vivienda en Iberoamérica destaca que en la mayoría de los países analizados las condiciones de una vivienda digna y sus carencias en materia de servicios continúan siendo un tema presente, dada la heterogeneidad y desigualdad social que existe (Garay Villegas et al., 2015). Las autoras señalan la importancia de considerar el espacio disponible en las viviendas, como así también la accesibilidad para facilitar el desempeño de actividades básicas en el interior y en el entorno próximo. En perspectiva comparada, la disponibilidad de información de España permite ver la falta de datos en los países de la región, que si bien se ha ido contemplando dimensiones de gran importancia todavía resta incorporar algunas temáticas claves para ampliar la visión de habitabilidad de la vivienda (Garay Villegas et al., 2015). 


\section{FUENTES Y MÉTODOS}

\section{Fuentes de información}

En este artículo se trabaja fundamentalmente a partir del análisis de los microdatos en formato Redatam de los censos de población, específicamente los Censos Nacionales de Población, Vivienda y Hogares de 1991, 2001 y 2010 relevados por el Instituto Nacional de Estadística y Censos (INDEC). La información censal constituye la fuente por excelencia para trabajar con altos grados de desagregación geográfica y, de esta forma, captar las especificidades que caracterizan las actuales dinámicas espaciales de la población adulta mayor.

La escala de desagregación utilizada es la menor unidad de superficie en que los datos censales se encuentran disponibles: los radios censales. En el caso de la ciudad de Córdoba, el número promedio de habitantes por radio es de 1.240, 927 y 871 personas para los censos de 1991, 2001 y 2010, respectivamente; aunque, se destaca una significativa variabilidad dependiendo si se tratan de subunidades espaciales ubicadas en las áreas centrales con una alta concentración de personas o si se encuentran localizadas en la periferia menos densa ${ }^{3}$.

Respecto al criterio de diferenciación para identificar a la población adulta mayor, en este trabajo se considera como adulto mayor a todas las personas que al momento del Censo tenían 60 años o más de edad y residían en viviendas particulares de la ciudad de Córdoba.

\section{Medidas de segregación e identificación de conglomerados espaciales}

La literatura especializada ha propuesto un gran número de índices para cuantificar la segregación residencial y, como destacan Kaztman y Retamoso (2009), cada uno enfatiza un aspecto o un grupo de aspectos del fenómeno, tales como la uniformidad en la distribución de los grupos en el espacio urbano (tales como el índice de disimilitud de Duncan, el índice de segregación residencial), el grado de contacto potencial entre personas de un mismo grupo o entre miembros de grupos diferentes (tales como el índice de aislamiento o de exposición), o el grado de contigüidad espacial entre unidades territoriales con características similares (tales como el índice de autocorrelación espacial de Moran).

Particularmente en este trabajo se optó por el Índice de Moran que evalúa la existencia de conglomerados en la distribución espacial de una variable, pudiendo ser calculado como medida general (Global) o de forma localizada para cada unidad espacial (Local). El Índice de Moran Global (I de Moran Global) constituye un estadístico resumen del grado de autocorrelación espacial y sintetiza en un coeficiente - la pendiente de la recta regresión- el grado de asociación entre un nivel dado en la variable de interés en un área geográfica respecto del promedio ponderado de la misma variable en las áreas contiguas o vecinas. Sus valores oscilan entre -1 y 1, es decir, desde una autocorrelación negativa perfecta (perfecta dispersión) a una autocorrelación positiva perfecta (perfecta concentración), donde 0 significa un patrón espacial completamente aleatorio (Anselin, 1993; 1995).

Sin embargo, este indicador, como cualquier otro índice global de autocorrelación espacial, no es capaz de detectar las estructuras locales de asociación o inestabilidades locales que pueden estar, a su vez, presentes o no en una estructura global de dependencia.
En cambio, aquellas medidas desarrolladas desde una perspectiva local, como la versión local del Índice de Moran, permiten explorar visualmente los patrones de aglomeración formados en función a los valores de una variable que presentan las unidades de observación analizadas y las unidades vecinas, verificándose la magnitud de la contribución de cada unidad espacial a la formación del valor general. Como resultado se obtiene un mapa, donde cada unidad espacial se diferencia de acuerdo al tipo de autocorrelación espacial que posee en relación a sus unidades espaciales vecinas (Anselin, 1993, 1995).

Para el cálculo del Índice de Moran Global y la generación de los mapas correspondientes se trabaja con la versión 10.3 de ArcGIS.

\section{Indicadores relativos a las condiciones de habitabilidad de las viviendas de los adultos mayores}

Para la evaluación de las condiciones de habitabilidad de las viviendas en las que reside al menos un adulto mayor en el espaciourbano de la ciudad de Córdoba, se seleccionaron cuatro indicadores (Cuadro 1).

\section{RESULTADOS}

\section{Distribución espacial de la población adulta mayor}

En la ciudad de Córdoba el proceso de envejecimiento demográfico se evidencia en el incremento, tanto absoluto como relativo, de la población de 60 años y más en los últimos 25 años. En 1991 el número de adultos mayores era de 138.552 efectivos, incrementándose a 166.848 en 2001 y a 191.113 en 2010. En términos porcentuales, ese grupo poblacional pasó de 11,7\% a 14,4\% entre 1991 y 2010 (Cuadro 2).

Asimismo, se observa también un aumento en el número absoluto y relativo de hogares particulares en los que reside al menos un adulto mayor: mientras en 1991 el 31,1\% de los hogares particulares estaba constituido por al menos una persona de 60 años y más, en 2010 ese porcentaje se elevó al 33,0\% (Cuadro 2).

Respecto a la localización espacial de los adultos mayores en el espacio residencial de la ciudad de Córdoba, se observan importantes diferencias en su distribución relativa en los radios censales (Figura 1). Si bien gran parte de este patrón de localización se corresponde con el patrón típico urbano donde las mayores proporciones de este grupo etario se concentran en las áreas centrales, se observan máximas de concentración en barrios pericentrales y periféricos del sector oeste. De acuerdo a Peralta y Liborio (2014), estos barrios vienen experimentando un proceso de despoblamiento, el cual se produce porque la ciudad expulsa población en un proceso centrífugo de población hacia la periferia o hacia las ciudades del área metropolitana.

La situación en 1991 muestra que el micro y macro centro de la ciudad registra las máximas concentraciones de adultos mayores (entre el $18 \%$ y el $33 \%$ ). Aunque también se observa una importante agrupación de este grupo poblacional en los radios envolventes al área central, principalmente en los barrios pericentrales e intermedios, tanto en los barrios conocidos como barrios-pueblo del siglo XIX (Alberdi, Alta Córdoba, Alto Alberdi, General Paz, San Martín, San Vicente, entre otros), como en los barrios tipo jardín de la década del '45 - 50 (Cerro de las Rosas, Cofico, Juniors, Los Naranjos, Maipú Primera Sección, Parque Vélez Sarsfield, Villa Cabrera, entre otros). 


\begin{tabular}{|c|c|c|}
\hline DIMENSIÓN & INDICADOR & DESCRIPCIÓN \\
\hline $\begin{array}{l}\text { Disponibilidad de servi- } \\
\text { cios, materiales, facilida- } \\
\text { des e infraestructura }\end{array}$ & $\begin{array}{l}\text { Porcentaje de hogares } \\
\text { según la calidad de las } \\
\text { conexiones de la vivienda a } \\
\text { servicios básicos. }\end{array}$ & $\begin{array}{l}\text { Refiere al tipo de instalaciones con que cuentan las viviendas para su sanea- } \\
\text { miento: procedencia del agua y tipo de desagüe. Las categorias son: } \\
\text { - Calidad satisfactoria: refiere a las viviendas que disponen de agua a red } \\
\text { pública y desagüe cloacal. } \\
\text { - Calidad básica: describe la situación de aquellas viviendas que disponen } \\
\text { de agua de red pública y el desagüe a pozo con cámara séptica. } \\
\text { - Calidad insuficiente: engloba a las viviendas que no cumplen ninguna de } \\
\text { las } 2 \text { condiciones anteriores. }\end{array}$ \\
\hline \multirow[t]{2}{*}{ Adecuación de la vivienda } & $\begin{array}{l}\text { Porcentaje de hogares se- } \\
\text { gún la calidad constructiva } \\
\text { de la vivienda. }\end{array}$ & $\begin{array}{l}\text { Refiere a la calidad de los materiales con los que está construida la vivienda } \\
\text { y las instalaciones internas a servicios básicos (agua de red y desagüe) de } \\
\text { las que dispone. Las categorías son: } \\
\text { - Calidad satisfactoria: refiere a las viviendas que disponen de materiales } \\
\text { resistentes, sólidos y con la aislación adecuada. A su vez también disponen } \\
\text { de cañerías dentro de la vivienda y de inodoro con descarga de agua. } \\
\text { - Calidad básica: no cuentan con elementos adecuados de aislación o } \\
\text { tienen techo de chapa o fibrocemento. Al igual que el anterior, cuentan con } \\
\text { cañerías dentro de la vivienda y de inodoro con descarga de agua. } \\
\text { - Calidad insuficiente: engloba a las viviendas que no cumplen ninguna de } \\
\text { las } 2 \text { condiciones anteriores. }\end{array}$ \\
\hline & $\begin{array}{l}\text { Porcentaje de hogares con } \\
\text { hacinamiento por cuarto. }\end{array}$ & $\begin{array}{l}\text { Representa el cociente entre la cantidad total de personas del hogar y la } \\
\text { cantidad total de habitaciones o piezas de que dispone el mismo (sin contar } \\
\text { baño/s y cocina/s). Se considera que un hogar presenta hacinamiento por } \\
\text { cuarto cuando el cociente da superior a tres. }\end{array}$ \\
\hline Arreglos residenciales & $\begin{array}{l}\text { Porcentaje de hogares con } \\
\text { personas mayores según } \\
\text { tipo de hogar y familia }\end{array}$ & $\begin{array}{l}\text { Se construye una tipología de hogares y familias, que para efectos de } \\
\text { análisis se ha definido de acuerdo a las características de la estructura de } \\
\text { parentesco de sus miembros respecto de quien se declara como jefe del } \\
\text { hogar, con las siguientes categorías: } \\
\text { a. unipersonales (una sola persona); y } \\
\text { b. sin núcleo (aquellos donde no existe un núcleo conyugal, es decir, una re- } \\
\text { lación padre/madre e hijo/hija, aunque puede haber otros lazos familiares). } \\
\text { Entre los tipos de familia se distinguen: } \\
\text { a. nucleares (padre o madre o ambos, con o sin hijos); } \\
\text { b. extendidas (padre o madre o ambos, con o sin hijos y otros parientes); } \\
\text { c. compuestas (padre o madre o ambos, con o sin hijos, con o sin otros } \\
\text { parientes y otros no parientes, excluyendo el servicio doméstico puertas } \\
\text { adentro y sus familiares). }\end{array}$ \\
\hline
\end{tabular}

$>$ Cuadro 1. Dimensiones e indicadores relativos a las condiciones de habitabilidad de las viviendas. Fuente: Elaboración propia con base en CELADE (2006) e INDEC (2013).

\begin{tabular}{|c|c|c|c|c|}
\hline & 1991 & 2001 & 2010 & $\begin{array}{c}\text { Variación porcentual } \\
1991-2010\end{array}$ \\
\hline Adultos mayores & 138.552 & 166.848 & 191.113 & 37,9 \\
\hline Total de personas & 1.179.372 & 1.284 .582 & 1.329 .604 & 12,7 \\
\hline Porcentaje de adultos mayores & 11,7 & 13,0 & 14,4 & \\
\hline Hogares particulares con al menos un adulto mayor & 90.117 & 118.362 & 136.880 & 51,9 \\
\hline Total de hogares particulares & 289.622 & 359.404 & 414.845 & 43,2 \\
\hline Porcentaje de hogares particulares con al menos un adulto mayor & 31,1 & 32,9 & 33,0 & \\
\hline
\end{tabular}

> Cuadro 2. Población adulta mayor (absoluta y relativa) en la ciudad de Córdoba. Años 1991, 2001 y 2010. Fuente: Elaboración propia con base en microdatos de los Censos de 1991, 2001 y 2010. 


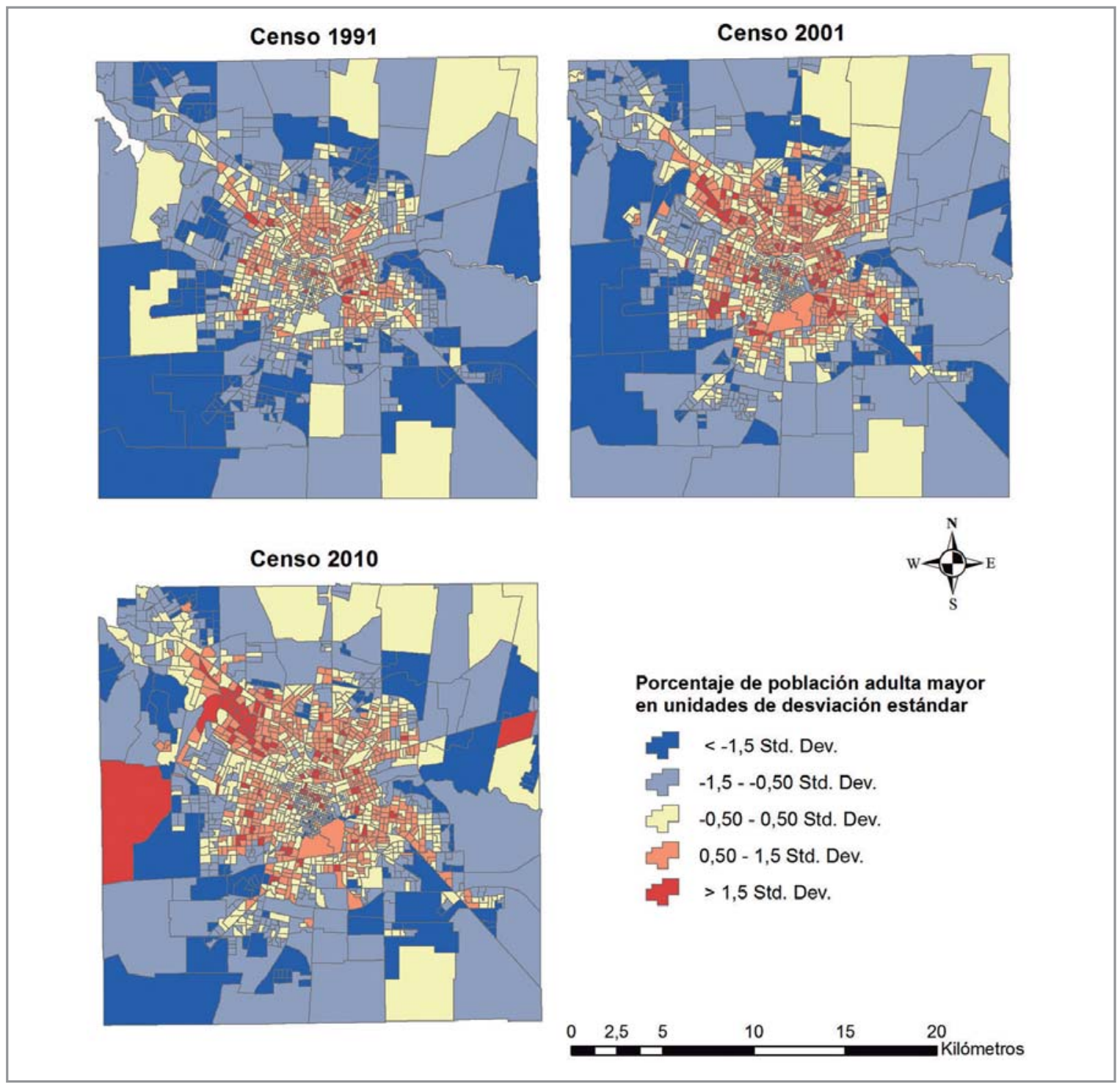

En cambio, en los barrios localizados en la periferia de la ciudad particularmente en los barrios que han absorbido planes de vivienda del gobierno o de cooperativas (Mauller, Quintas de Argüello; Ramón J. Cárcano, Mirador, Ferrer, San Javier, Los Filtros, Cerveceros, BialetMasse. San Marcelo, Rosedal, Colonia Lola y El Trébol, etc) o en los barrios jardín periféricos que han absorbido fuerte inversión inmobiliaria (Villa Belgrano, Las Rosas)-, el porcentaje de adultos mayores es significativamente inferior, observándose porcentajes muy por debajo de la media local (entre el 0,4 y el 6\%).

Para 2001 si bien se observan continuidades en los patrones de localización espacial de la población de 60 años y más, también se registra un mayor número de radios censales con altas concentraciones de este grupo poblacional. Es decir, por un lado, se mantienen las máximas de concentración en el micro y macro centro y en los barrios que lo envuelven, y las mínimas en la periferia de la ciudad. Pero, por otro lado, se observa la inclusión de un mayor número de radios censales ubicados en el área pericentral y la inclusión de otros localizados en la periferia noroeste de la ciudad con altos porcentajes de adultos mayores (por ejemplo, Argüello y Parque Jorge Newberry).

$>$ Figura 1. Distribución relativa de la población adulta mayor en unidades de desviación estándar. Ciudad de Córdoba. Años 1991, 2001 y 2010.

Fuente: Elaboración propia con base en microdatos de los Censos de 1991, 2001 y 2010. 
En 2010 se evidencia una profundización de los cambios observados entre 1991 y 2001, principalmente en lo referido a la presencia de máximas de concentración en los barriospericentrales y periféricos localizados en el corredor noroeste de la ciudad (tales como, Argüello, Cerro de las Rosas, Marques de Sobremonte, Parque Tablada, Quebrada de las Rosas, Villa Cabrera, Villa Centenario, Urca).

\section{¿Cuán segregada está la población adulta mayor?}

Los resultados del I de Moran Global obtenidos para la ciudad de Córdoba para los años 1991, 2001 y 2010 sugieren la presencia de altos niveles de segregación residencial en la población adulta mayor y la presencia de dependencia espacial positiva entre los radios censales. Entre 1991 y 2010 los valores de I de Moran Global presentan significativas disminuciones; aunque, se mantiene la presencia de autocorrelación global positiva observada a comienzos de la última década del siglo XX (Cuadro 3).

\begin{tabular}{l|r|r|r}
\hline & \multicolumn{1}{|c|}{1991} & \multicolumn{1}{c|}{2001} & \multicolumn{1}{c}{2010} \\
\hline I de Moran Global & 0,6352 & 0,6284 & 0,5561 \\
\hline Índice esperado & $-0,000888$ & $-0,000728$ & $-0,000670$ \\
\hline Varianza & 0,000299 & 0,000244 & 0,000217 \\
\hline Puntuación z & 36,8007 & 40,2368 & 37,8168 \\
\hline Valor p & 0,0000 & 0,0000 & 0,0000 \\
\hline
\end{tabular}

$>$ Cuadro 3. Estadísticos descriptivos. Índice Global de Moran para población adulta mayor en la ciudad de Córdoba. Años 1991, 2001 y 2010. Nota: Valores p-seudo significativos al 0,001. Para todos los casos se rechaza hipótesis nula de aleatoriedad. Fuente: Elaboración propia con base en microdatos de los Censos de 1991, 2001 y 2010.

En 1991 la intensidad de la segregación fue aproximadamente un $14 \%$ más alta si la comparamos con la registrada en el 2010, a pesar de que la población envejecida creció en el mismo periodo en más de $27 \%$ (cerca de 52.000 nuevos adultos mayores). A pesar de los retrocesos en los indicadores de segregación residencial, los adultos mayores se encuentran altamente segregados en el espacio residencial de la ciudad de Córdoba.

Estudios realizados para el área metropolitana de Toluca para los años 1990 y 2000, (Garrocho y Campos, 2005) y la Ciudad de México, años 2000 y 2010 (Garrocho y Campos, 2015) también evidencian altos niveles de segregación en la población adulta mayor (65 años y más) y observándose un retroceso, muy leve en el caso de Toluca, a lo largo del período analizado. En términos comparativos, la ciudad de Córdoba presenta valores intermedios a los observados en las otras dos ciudades.

La disminución marginal del I de Moran Global podría explicarse por el proceso de expansión urbana de la ciudad de Córdoba, que si bien no se tradujo en la incorporación de nuevas superficies totales ya que no se ha modificado la superficie total del municipio, sino en la urbanización de nuevas superficies y en la creación de nuevos barrios residenciales. También entran en juego otros factores como los procesos de despoblamiento, deterioro y cambio de rol que han sufrido los diferentes barrios de la ciudad desde 1970 y que se han acelerado en los últimos 25 años, vinculados al cambio socio-demográfico. Por ejemplo, se observa la gentrificación de ciertas áreas pericentrales (Alta Córdoba, Cofico, General Paz o Juniors), que recibieron población joven de ingresos medios altos y altos; la pérdida paulatina de población envejecida en ciertas partes tradicionales de la ciudad al concluir su ciclo de vida, entre otros factores (Peralta y Liborio (2014).

\section{¿Dónde se concentra espacialmente la población adulta mayor?}

La variante local del I de Moran permite identificar, a partir de la desagregación de la versión global del índice, las zonas específicas de la ciudad donde se concentran los adultos mayores y clasificar las unidades territoriales -en este caso, los radios censales- en cuatro categorías estadísticamente significativas. Las categorías Alto - Alto y Bajo - Bajo (identificadas los mapas en rojo y en azul, respectivamente) constituyen agrupamientos o clusters territoriales, dado que están conformados por radios censales con valores similares entre sí. Por ejemplo, los radios censales con alta presencia de población envejecida rodeada de otros radios que también señalan altos porcentajes de adultos mayores, conforman agrupamientos clasificados como Alto - Alto y, por lo tanto, se trata de clusters territoriales envejecidos.

Las otras dos categorías que se obtienen se integran por radios censales con valores altos o bajos de envejecimiento, que colindan con vecinos que exhiben valores bajos o altos, respectivamente: por ejemplo, la categoría Alto - Bajo está integrada por radios censales con una importante presencia relativa de adultos mayores, rodeados de unidades territoriales con muy bajo o bajo porcentaje de población envejecida. Ambas categorías permiten identificar zonas de aislamiento donde se están gestando procesos importantes en términos de la recomposición demográfica.

En la ciudad de Córdoba predominan, en los tres años censales considerados, la presencia de clusters territoriales: mientras los radios censales clasificados como Alto - Alto representan agrupamientos espaciales envejecidos, los clasificados como Bajo - Bajo indican lo contrario. Como puede visualizarse en la Figura 2, los conglomerados Alto - Alto se localizan predominantemente en las áreas centrales y en los barrios pericentrales. Estos patrones espaciales se mantienen en gran medida en el período analizado; aunque, se observa hacia 2010 una menor presencia de estos conglomerados en los barrios pericentrales y la consolidación de un cluster envejecido en el corredor noroeste de la ciudad. Este cluster, además de presentar una alta concentración de adultos mayores, coincide parcialmente con las zonas caracterizadas por una "segregación por riqueza" en otro trabajo realizada también para la ciudad de Córdoba (Molinatti, 2013). De acuerdo a Peralta y Liborio (2014), los barrios ubicados en este corredor se caracterizan por un marcado proceso de despoblamiento, con pérdidas de población de alrededor del 40\% en los últimos 25 años.

Condiciones de habitabilidad de las viviendas en las áreas con alta concentración espacial de población adulta mayor

A partir de la identificación de los conglomerados espaciales donde se observa una alta concentración de adultos mayores, se analizaron comparativamente las condiciones de habitabilidad de las viviendas en las que residían hogares con al menos un adulto mayor. La habitabilidad fue abordada a partir de tres indicadores claves: acceso a servicios básicos, adecuación de la vivienda y hacinamiento por cuarto.

- Acceso a servicios básicos. De la comparación los resultados de este indicador, fundamental para asegurar la salud de las adultos mayores que residen en la vivienda, se desprende que entre las diferentes áreas residenciales donde se concentran este grupo poblacional existen importantes brechas. En el área central y los barrios pericentrales casi no se registran hogares en viviendas que no disponen de agua por red 


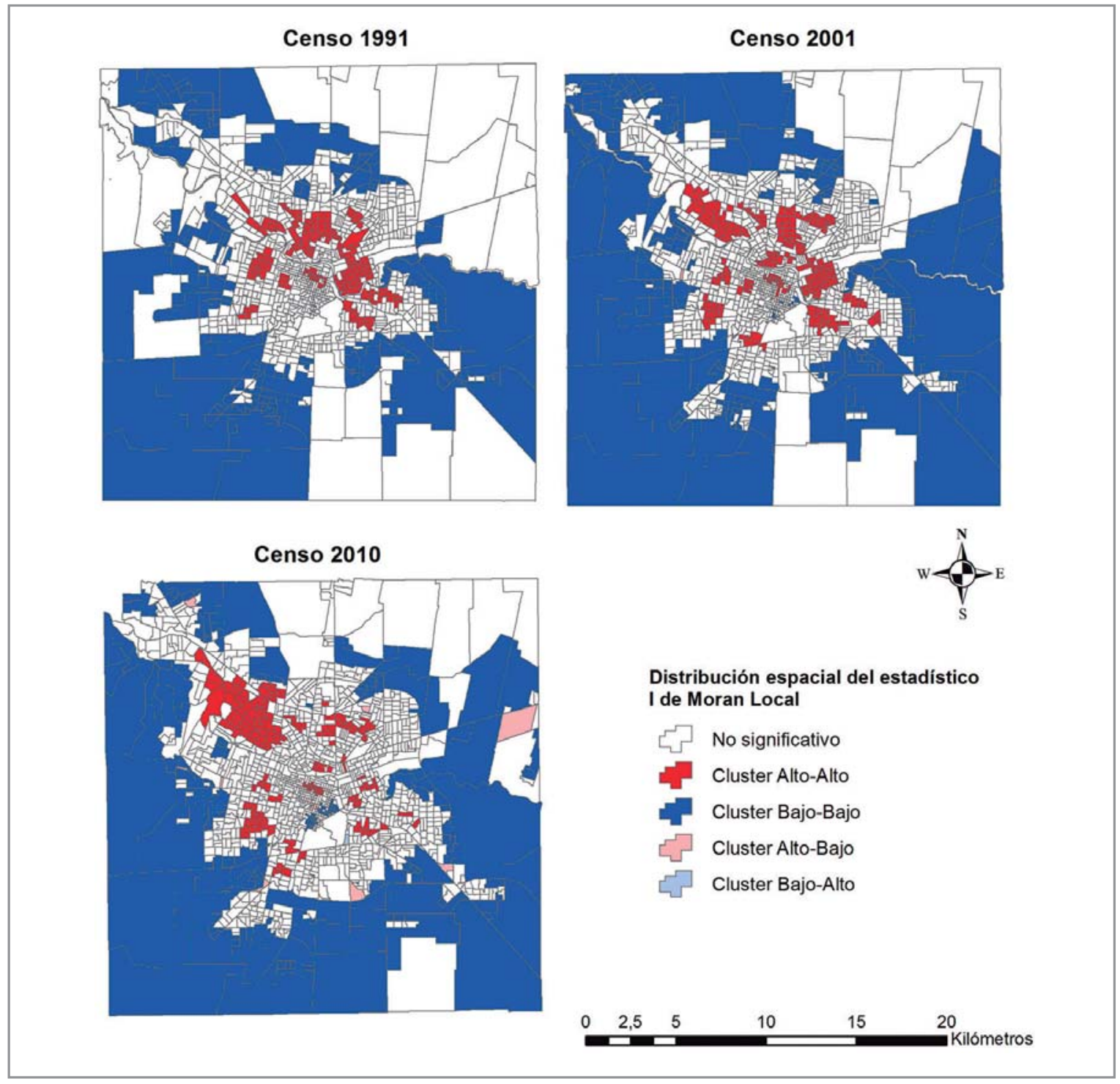

o desagüe cloacal (calidad satisfactoria). En cambio, en los barrios ubicados en el segundo anillo intermedio y el anillo periférico, si bien es mayor el porcentaje de hogares en viviendas con calidad satisfactoria en las conexiones, se observa un importante porcentaje de hogares en viviendas que disponen de agua por red pero no cuentan con desagüe cloacal. La mayor brecha se observa entre el área central donde casi la totalidad de los hogares tienen una calidad satisfactoria en las conexiones de la vivienda a los servicios básicos y los barrios ubicados en la zona suroeste del anillo periférico (Colinas, Parque y Residencial Vélez Sarsfield, entre otros) donde el porcentaje se reduce al $88 \%$ (Cuadro 4).

- $\quad$ Adecuación de la vivienda. El porcentaje de hogares con al menos un adulto mayor en viviendas con calidad constructiva satisfactoria presenta una menor variabilidad entre los diferentes conglomerados residenciales a la observada en el acceso a servicios básicos; aunque, también para este indicador el área central exhibe el mayor porcentaje de hogares en viviendas con calidad satisfactoria (aproximadamente 7 de cada 10 hogares). En los barrios del segundo anillo inter-

$>$ Figura 2. Distribución espacial del estadístico I de Moran Local para la población adulta mayor. Ciudad de Córdoba. Años 1991, 2001 y 2010. Fuente: Elaboración propia con base en microdatos de los Censos de 1991, 2001 y 2010. 


\begin{tabular}{|c|c|c|c|c|}
\hline & SATISFACTORIO & BÁsICO & INSUFICIENTE & TOTAL HOGARES \\
\hline Área Central y Alberdi & 99,0 & 0,1 & 0,4 & 1.543 \\
\hline \multicolumn{5}{|l|}{ BARRIOS PERICENTRALES } \\
\hline Juniors, General Paz y otros & 97,2 & 2,2 & 0,5 & 1.093 \\
\hline Cofico y Alta Córdoba (sur) & 98,9 & 0,4 & 0,4 & 529 \\
\hline Maipú $1^{\circ}$ y $2^{\circ}$ Sección, Colón y otros & 91,0 & 6,4 & 2,4 & 901 \\
\hline \multicolumn{5}{|l|}{ Segundo anillo intermedio } \\
\hline Ayacucho, Residencial América y Alta Córdoba (norte) & 78,2 & 19,0 & 2,7 & 2.337 \\
\hline A.M. Zumarán y Los Paraísos & 91,0 & 7,0 & 2,0 & 686 \\
\hline \multicolumn{5}{|l|}{ ANILLO PERIFÉRICO } \\
\hline Colinas, Parque y Residencial Vélez Sarsfield y otros & 42,2 & 46,2 & 11,6 & 1.306 \\
\hline Los Naranjos, San Rafael, Rosedal y otros & 62,3 & 33,6 & 3,9 & 2.267 \\
\hline Corredor Noroeste & 66,1 & 30,2 & 3,2 & 7.371 \\
\hline TOTAL & 73,3 & 23,1 & 3,3 & 18.033 \\
\hline
\end{tabular}

$>$ Cuadro 4. Porcentaje de hogares particulares con al menos un adulto mayor según calidad de las conexiones de la vivienda a los servicios básicos. Áreas seleccionadas de la ciudad de Córdoba, 2010. Fuente: Elaboración propia con base en microdatos del Censo de 2010.

medio, en cambio, se observa que esa proporción se reduce a uno de cada dos. La mayor brecha entre zonas se registra entre el área central y los barrios Ana María Zumarán y Los Paraísos ubicados en la zona norte del segundo anillo intermedio: el porcentaje de hogares en viviendas de calidad constructiva satisfactoria en el área central supera en un $50 \%$ al porcentaje obtenido para los otros dos barrios (Cuadro 5).

- Hacinamiento. El porcentaje de hogares con hacinamiento crítico (más de tres personas por cuarto) no alcanza al 0,5\% en ninguno de los conglomerados espaciales identificados. Sin embargo, si se incluye a los hogares con hacinamiento moderado (más de dos personas por cuarto) aumenta significativamente el porcentajes de hogares con al menos un adulto mayor hacinados y las diferencias entre conglomerados son más visibles. El conglomerado con menor nivel de hacinamiento es el localizado en el corredor noroeste, con el $6 \%$ de los hogares con más de 2 personas por cuarto; en contraste, los mayores niveles de hacinamiento se registran en el cluster conformado por los barrios Zumarán y Los Paraísos en la zona norte de la ciudad, donde casi el $17 \%$ de los hogares presenta hacinamiento moderado o crítico (Cuadro 6).

\begin{tabular}{|c|c|c|c|c|}
\hline & SATISFACTORIO & BÁSICO & INSUFICIENTE & TOTAL HOGARES \\
\hline Área Central y Alberdi & 72,8 & 26,4 & 0,3 & 1.543 \\
\hline \multicolumn{5}{|l|}{ BARRIOS PERICENTRALES } \\
\hline Juniors, General Paz y otros & 68,3 & 30,7 & 0,8 & 1.093 \\
\hline Cofico y Alta Córdoba (sur) & 73,0 & 25,7 & 0,9 & 529 \\
\hline Maipú $1^{\circ}$ y $2^{\circ}$ Sección, Colón y otros & 64,8 & 33,4 & 1,7 & 901 \\
\hline \multicolumn{5}{|l|}{ SEGUNDO ANILLO INTERMEDIO } \\
\hline Ayacucho, Residencial América y Alta Córdoba (norte) & 57,1 & 41,8 & 0,9 & 2.337 \\
\hline A.M. Zumarán y Los Paraísos & 48,1 & 50,3 & 1,6 & 686 \\
\hline \multicolumn{5}{|l|}{ ANILLO PERIFÉRICO } \\
\hline Colinas, Parque y Residencial Vélez Sarsfield y otros & 54,1 & 45,3 & 0,5 & 1.306 \\
\hline Los Naranjos, San Rafael, Rosedal y otros & 66,3 & 32,7 & 0,8 & 2.267 \\
\hline Corredor Noroeste & 68,1 & 30,8 & 0,6 & 7.371 \\
\hline TOTAL & 65,1 & 33,8 & 0,8 & 18.033 \\
\hline
\end{tabular}

$>$ Cuadro 5. Porcentaje de hogares particulares con al menos un adulto mayor según calidad constructiva de la vivienda. Áreas seleccionadas de la ciudad de Córdoba, 2010. Fuente: Elaboración propia con base en microdatos del Censo de 2010.

16 > Revista Márgenes № 19 Vol 13 > Diciembre 2016: 7 a 20 Facultad de Arquitectura > Universidad de Valparaíso 
Si comparamos los hogares con por al menos un adulto mayor con aquellos no envejecidos, se observa que los primeros presentan menores niveles de hacinamiento que los segundos y una mayor variabilidad entre los conglomerados residenciales. En los diferentes clusters analizados se observa un patrón común: el porcentaje de hogares no envejecidos con algún nivel de hacinamiento casi triplica al registrado en los hogares envejecidos, evidenciándose en estos últimos hogares mejores condiciones de habitabilidad en lo referido a la relación persona-cuarto. (Cuadro 6).

- Arreglos residenciales. Además de las condiciones de habitabilidad de las viviendas, otro aspecto que reviste particular importancia son los arreglos residenciales de las personas mayores, ya que el hogar es uno de los ámbitos más importantes para las transferencias de apoyo.

\begin{tabular}{|c|c|c|c|c|c|c|c|c|}
\hline & \multicolumn{4}{|c|}{ CON AL MENOS UN ADULTO MAYOR } & \multicolumn{4}{|c|}{ SIN ADULTOS MAYORES } \\
\hline & No & Moderado & Crítico & TOTAL & No & Moderado & Crítico & TOTAL \\
\hline Área Central & 91,8 & 8,1 & 0,1 & 1.543 & 81,7 & 18,2 & 0,1 & 4.028 \\
\hline \multicolumn{9}{|l|}{ BARRIOS PERICENTRALES } \\
\hline Juniors, General Paz y otros & 92,0 & 7,8 & 0,2 & 1.093 & 83,5 & 16,3 & 0,2 & 2.428 \\
\hline Cofico y Alta Córdoba (sur) & 84,1 & 15,7 & 0,2 & 529 & 82,7 & 17,1 & 0,2 & 1.912 \\
\hline Maipú $1^{\circ}$ y $2^{\circ}$ Sección, Colón y otros & 95,9 & 4,0 & 0,1 & 901 & 85,0 & 14,8 & 0,2 & 1.301 \\
\hline \multicolumn{9}{|l|}{ SEGUNDO ANILLO INTERMEDIO } \\
\hline Ayacucho, Residencial América y Alta Córdoba (norte) & 88,3 & 11,3 & 0,3 & 2.337 & 78,7 & 20,8 & 0,5 & 4.872 \\
\hline A.M. Zumarán y Los Paraísos & 83,4 & 16,2 & 0,4 & 686 & 71,9 & 27,0 & 1,1 & 1.474 \\
\hline \multicolumn{9}{|l|}{ ANILLO PERIFÉRICO } \\
\hline Colinas, Parque y Residencial Vélez Sarsfield y otros & 90,0 & 9,8 & 0,2 & 1.306 & 84,2 & 15,5 & 0,4 & 2.527 \\
\hline Los Naranjos, San Rafael, Rosedal y otros & 90,7 & 9,2 & 0,1 & 2.267 & 81,8 & 17,8 & 0,4 & 4.644 \\
\hline Corredor Noroeste & 94,0 & 5,9 & 0,1 & 7.371 & 88,5 & 11,5 & 0,1 & 14.991 \\
\hline TOTAL & 91,7 & 8,2 & 0,1 & 18.033 & 84,0 & 15,7 & 0,3 & 38.177 \\
\hline
\end{tabular}

$>$ Cuadro 6. Porcentaje de hogares particulares según nivel de hacinamiento por cuarto y presencia de adultos mayores. Áreas seleccionadas de la ciudad de Córdoba, 2010.

Mientras en el área central uno de cada dos hogares particulares con al menos un adulto mayor está compuesto por una sola persona, en el resto de los conglomerados espaciales esa relación se reduce aproximadamente a uno de cada cuatro, observándose el menor porcentaje de hogares unipersonales en el cluster conformado por los barrios Colinas, Parque y Residencial Vélez Sarsfield y otros (Cuadro 7).

Los hogares nucleares, con y sin pareja, con hijos, por otra parte, predominan en los conglomerados residenciales localizados en el anillo periférico; aunque se destaca que los barrios pericentrales Maipú $1^{\circ}$ y $2^{\circ}$ sección, Colón y otros poco más de un cuarto de los hogares con al menos un adulto mayor cuenta con hijo/s residiendo con uno o ambos pares mayores (Cuadro 7).

Los hogares extendidos, en cambio, tienen mayor presencia en los conglomerados que se ubican en el segundo anillo intermedio de la ciudad, en los cuales el 30\% de los hogares con al menos un adulto mayor se componen por la familia nuclear y otros familiares (Cuadro 7).

Respecto a los porcentajes de hogares compuestos y otros (no familiares, sólo con servicio doméstico y otros), se observa que en todos los conglomerados los porcentajes son inferiores a $5 \%$, observándose el mayor número de hogares compuestos en los agrupamientos en barrios pericentrales: uno en los barrios de Cofico y Alta Córdoba (zona sur), y otro en los barrios Maipú $1^{\circ}$ y $2^{\circ} \mathrm{sec}-$ ción, Colón y otros. En el área central y el corredor noroeste, por su parte, predominan los hogares compuestos por no familiares o por un adulto mayor y el servicio doméstico (Cuadro 7). 


\begin{tabular}{|c|c|c|c|c|c|c|c|}
\hline & Unipersonal & $\begin{array}{c}\text { Con } \\
\text { pareja }\end{array}$ & $\begin{array}{c}\text { Nuclear } \\
\text { con } \\
\text { hijo/s }\end{array}$ & Extendido & Compuesto & Otro & $\begin{array}{c}\text { Total } \\
\text { hogares }\end{array}$ \\
\hline Área Central y Alberdi & 49,2 & 19,6 & 12,7 & 14,1 & 1,0 & 3,4 & 1.543 \\
\hline \multicolumn{8}{|l|}{ BARRIOS PERICENTRALES } \\
\hline Juniors, General Paz y otros & 28,3 & 18,8 & 10,2 & 25,3 & 0,6 & 0,1 & 1.093 \\
\hline Cofico y Alta Córdoba (sur) & 26,7 & 23,6 & 9,5 & 21,9 & 1,7 & 0,4 & 529 \\
\hline Maipú $1^{\circ}$ y $2^{\circ}$ Sección, Colón y otros & 24,0 & 22,2 & 25,5 & 24,9 & 1,6 & 1,9 & 901 \\
\hline \multicolumn{8}{|l|}{ SEGUNDO ANILLO INTERMEDIO } \\
\hline $\begin{array}{l}\text { Ayacucho, Residencial América y Alta Córdoba } \\
\text { (norte) }\end{array}$ & 22,9 & 21,9 & 23,4 & 29,8 & 0,9 & 1,1 & 2.337 \\
\hline A.M. Zumarán y Los Paraísos & 24,6 & 18,5 & 23,0 & 31,6 & 1,2 & 1,0 & 686 \\
\hline \multicolumn{8}{|l|}{ ANILLO PERIFÉRICO } \\
\hline $\begin{array}{l}\text { Colinas, Parque y Residencial Vélez Sarsfield y } \\
\text { otros }\end{array}$ & 20,7 & 25,7 & 26,0 & 24,1 & 1,8 & 1,8 & 1.306 \\
\hline Los Naranjos, San Rafael, Rosedal y otros & 22,0 & 23,2 & 27,3 & 24,9 & 0,9 & 1,8 & 2.267 \\
\hline Corredor Noroeste & 22,6 & 24,6 & 27,8 & 21,2 & 1,7 & 2,2 & 7.371 \\
\hline TOTAL & 25,3 & 23,0 & 25,0 & 23,2 & 1,4 & 2,1 & 18.033 \\
\hline
\end{tabular}

> Cuadro 7. Porcentaje de hogares particulares con al menos un adulto mayor según arreglo residencial. Áreas seleccionadas de la ciudad de Córdoba, 2010. Fuente: Elaboración propia con base en microdatos del Censo de 2010.

\section{CONCLUSIONES}

El envejecimiento de la población constituye el tema demográfico más importante que enfrenta la ciudad de Córdoba en el siglo XXI. El grupo de población de 60 años y más ha incrementado su volumen considerablemente en los últimos 25 años y a un ritmo mucho mayor al observado para el total de la población: mientras el número de adultos mayores aumentó en casi un 38\% entre 1991 y 2010, el total de la población experimentó un incremento significativamente menor y cercano al $13 \%$.

¿En qué medida las ciudades, incluida la ciudad de Córdoba, están preparadas para este acelerado proceso de envejecimiento en un contexto donde las ciudades alojan a gran parte de la población, incluida la envejecida? ¿Cuán segregados se encuentran los adultos mayores en términos residenciales? ¿Dónde se concentran espacialmente? ¿Cuán homogéneas son las condiciones de habitabilidad y los arreglos residenciales en esos conglomerados? Este trabajo procuró responder estas preguntas y tuvo como objetivo abordar la relación funcional entre la ciudad y la población adulta mayor en la ciudad de Córdoba, tomando como eje de análisis la segregación residencial de este grupo poblacional y las condiciones de habitabilidad de las viviendas en los conglomerados residenciales envejecidos.

Los resultados sugieren los adultos mayores muestran un patrón residencial bien definido, ocupando y apropiándose de zonas específicas del espacio urbano. En el período 1991 - 2010 la intensidad de la segregación ha disminuido; aunque, los valores del I de Moran Global indican aun en 2010 altos niveles de segregación residencial. Comparativamente con otras ciudades latinoamericanas, la ciudad de Córdoba presenta valores intermedios a los observados en el área metropolitana de Toluca (1990 y 2000) y la Ciudad de México (2000 y 2010) (Garrocho y Campos, 2005 y 2015).

En la última década del siglo XX las mayores concentraciones de la población adulta mayor se registraban en el micro y macro centro de la ciudad, con importantes volúmenes de población en algunos

18 > Revista Márgenes № 19 Vol 13 > Diciembre 2016: 7 a 20 Facultad de Arquitectura > Universidad de Valparaíso 
barrios pericentrales e intermedios (Alberdi, Alta Córdoba, Cerro de Ias Rosas, Cofico, General Paz, Juniors, Villa Cabrera, entre otros). Mientras que en la periferia de la ciudad no se observan conglomerados residenciales compuestos por altos porcentajes de adultos mayores, sino que, por el contrario, los barrios localizados en esa área se caracterizan por tener un perfil demográfico no envejecido.

A comienzos de la segunda década del siglo XXI, si bien se observan continuidades en los patrones de localización residencial de la población adulta mayor, también se profundizan los cambios manifestados a comienzos del siglo con la incorporación de nuevas superficies con altas concentraciones espaciales de este grupo poblacional, principalmente en el área pericentral y el corredor noroeste de la ciudad.

Considerando los antecedentes que indican una escasa movilidad residencial, aunque mayor en los países más avanzados en la transición demográfica, de los adultos mayores en una serie de países de la región (Pérez Amador y Brenes, 2006), los cambios observados en la localización espacial de los adultos mayores, podrían explicarse por otros procesos demográficos y urbanos que se han manifestado con fuerza en los últimos 25 años. Por un lado, se observa un proceso de deterioro y despoblamiento del área central y de los barrios tradicionales (Cerro de las Rosas, Colinas de Vélez Sarsfield, Los Naranjos, Maipú $1^{\circ}$ Sección, Villa Cabrera), y la consiguiente dispersión poblacional hacia nuevas áreas periféricas. Por otro lado, un proceso de gentrificación, reflejada en un "boom" inmobiliario sin precedentes, de ciertas áreas pericentrales (Alberdi, Alta Córdoba, General Paz, entre otros), las cuales recibieron población joven con ingresos medios altos y altos.

El análisis comparativo de las condiciones de habitabilidad sugiere que los conglomerados espaciales con máximas concentraciones de adultos mayores distan de ser homogéneos entre sí, observándose mejores condiciones en el área central, los barrios pericentrales y los barrios tradicionales localizados en el corredor noroeste de la ciudad. Estos conglomerados coinciden con las zonas de "segregación por riqueza" identificadas en otro trabajo sobre la segregación residencial, pero socioeconómica, realizado también para la ciudad de Córdoba (Molinatti, 2013).

En cambio, en los barrios del segundo anillo intermedio y otros barrios tradicionales del anillo periférico se registran porcentajes significativos de adultos mayores con acceso básico insuficiente a servicios básicos, viviendas con calidad constructiva que demanda refacciones, especialmente en lo referido al aislamiento térmico, y niveles moderados de hacinamiento (más de dos personas por cuarto). Estas zonas, si bien presentan peores condiciones de habitabilidad, corresponden a conglomerados con alta diversidad socioeconómica (Molinatti, 2013).

Respecto a los arreglos residenciales de los adultos mayores predominantes en los agrupamientos espaciales identificados, se observa que los hogares unipersonales o compuestos solamente por la pareja predominan en el área central, similar a lo observado en los países con un proceso de envejecimiento más avanzado; mientras que en los barrios del segundo anillo intermedio y aniIlo periférico los hogares nucleares con hijos y extendidos tienen una presencia importante, análogo a lo registrado en países con un envejecimiento incipiente o moderado (Peláez y Féliz Ferreras, 2010). Los resultados sugieren que si bien la ciudad de Córdoba se encuentra avanzada en su transición demográfica, al interior del espacio urbano se identifican agrupamientos en diferentes estadios del proceso.

En síntesis, la segregación residencial de los adultos mayores es un fenómeno presente en la ciudad de Córdoba y con grandes heterogeneidades como consecuencia de los procesos urbanos y demográficos que han moldeado y cambiado los perfiles de los espacios residenciales de la ciudad. En este sentido, si bien actualmente no existe un consenso sobre las ventajas y desventajas de este fenómeno socioespacial presente en gran parte de las ciudades del mundo, predominan los argumentos a favor de su integración en pos de la cohesión social, las interacciones significativas intergeneracionales y la disponibilidad de apoyo social.

Debido al carácter acelerado e irreversible del proceso de envejecimiento demográfico, la inclusión de la segregación residencial de los adultos mayores en los debates urbanos y en las políticas públicas resulta imprescindible, siendo el verdadero reto el diseño de políticas que ajusten los diseños de las ciudades a los requerimientos de este grupo poblacional y brinden la posibilidad a la población adulta mayor de envejecer en la comunidad ${ }^{4}$, como así también la creación de espacios intergeneracionales que promuevan la integración social.

\section{BIBLIOGRAFÍA}

ANDREWS, G., CUTCHINB, M., MCCRACKENC, K., PHILLIPS, D. R., WILES, J. (2007) "Geographical Gerontology: The constitution of a discipline", Social Science \& Medicine 65, pp. 151-168.

BOSH MEDA, J. (2006) “El problema de la vivienda en la vejez en Cataluña", ACE: Architecture, City and Environment 1(1), pp. 80-101.

BOSH MEDA, J. (2013) "Ciudad y envejecimiento: bases para un nuevo urbanismo: V Premio Nacional de Urbanismo Ricardo Santos Diez", Práctica urbanística: Revista mensual de urbanismo, 120, pp. 36-51.

BRUN, J. (1994) "Essai critique sur la notion de ségrégation et sur son usage en géographieurbaine", en Brun, J. y Rhein, C. (eds.), La ségrégationdans la ville. Concepts et mesures, pp. 21-57, Paris: L'Harmattan.

CELADE - Centro Latinoamericano y Caribeño de DemografíaDivisión de Población de la CEPAL (2006) Manual sobre indicadores de calidad de vida en la vejez (LC/W.113), Santiago: Comisión Económica para América Latina y el Caribe (CEPAL).

CEPAL - Comisión Económica para América Latina y el Caribe (2004) Población, envejecimiento y desarrollo, Trigésimo período de sesiones de la CEPAL, San Juan, Puerto Rico, 28 de junio al 2 de julio.

CEPAL (2011) "Envejecimiento poblacional", Observatorio demográfico América Latina y el Caribe 12.

FORMIGA, N., PRIETO, M. B. (2008) “Envejecimiento y diferenciación socio-espacial en calidad de vida. La situación en Bahía Blanca, Argentina", en Peláez, E. (org.), Sociedad y Adulto Mayor en América Latina: Estudios sobre Envejecimiento en la Región, pp. 39-67, Rio de Janeiro: Asociación Latinoamericana de Población. 
GARAY VILLEGAS, S., MONTES DE OCA ZAVALA, V., HEBRERO MARTíNEZ, M. (2015) "Los entornos y el envejecimiento en Iberoamérica: análisis a partir de las condiciones de la vivienda", Notas de Población 101, pp.109-125.

GARROCHO, C., CAMPOS, J. (2015). "Segregación socioespacial de la población mayor en la Ciudad de México, 2000 2010", en Consejo Nacional de Población, La Situación Demográfica de México 2015, pp. 167-195, Ciudad de México: CONAPO.

GARROCHO, C., CAMPOS, J. (2005) "La población adulta mayor en el área metropolitana de Toluca, 1990-2000". Papeles de Población, 11(45), pp. 71-106

GRAFMEYER, Y. (1994) “Regards sociologiques sur la ségrégation", en Brun, J. y Rhein, C. (eds.), La ségrégation dans la ville. Concepts et mesures, pp. 85-117, Paris: L'Harmattan.

HAGESTAD, G. y UHLENBERG, P. (2006) "Should We Be Concerned About Age Segregation? Some Theoretical and Empirical Explorations", Research on Aging 28(6), pp. 638-653.

INDEC (Instituto Nacional de Estadística y Censos) (2013) Censo Nacional de Población, Hogares y Viviendas 2010. Base de datos REDATAM. Definiciones de la base de datos, Buenos Aires: INDEC.

MOLINATII, F. (2013) “Segregación residencial socioeconómica en la ciudad de Córdoba (Argentina). Tendencias y patrones espaciales", Revista INVI 28(79), pp. 61-94.

NARVÁEZ MONTOYA, O. L. (2011) “Urbanismo Gerontológico: Envejecimiento demográfico y equipamiento urbano en Aguascalientes", Investigación y Ciencia 19(51), pp. 16-24.

NEGRETE SALAS, M. E. (2003) “El envejecimiento poblacional en la Ciudad de México: evolución y pautas de distribución espacial entre 1970 y 2000", Papeles de Población 9(37), pp. 1-21.

OMS (Organización Mundial de la Salud) Informe mundial sobre el envejecimiento y la salud, Estados Unidos de América: OMS.

PELÁEZ, E., FÉLIZ-FERRERAS, J. (2010) “Transición demográfica y arreglos residenciales de los adultos mayores en República Dominicana y Argentina", Papeles de Población 16(63), pp. 85-115.

PELÁEZ, E. (2006) “Selectividad residencial de los adultos mayores en la ciudad de Córdoba", Astrolabio (3), pp. 1-16.

PELÁEZ, E. (2005) "Selectividad residencial y vulnerabilidad de los adultos mayores en la ciudad de Córdoba. Magnitud, características y evolución", trabajo presentado en las VIII Jornadas de la Asociación de Estudios de Población de la Argentina, Tandil, Argentina, 12 al 14 de octubre.

PERALTA, C., LIBORIO, M. (2014) "Redistribución poblacional en la ciudad de Córdoba entre los períodos intercensales 1991 - 2001 / 2001 - 2008. Evaluación de los procesos de dispersión, densificación, gentrificación y renovación", Revista Vivienda y Ciudad 1, pp. 99-113.

PÉREZ AMADOR, J., BRENES, G. (2006) “Una transición en edades avanzadas: cambios en los arreglos residenciales de adultos mayores en siete ciudades latinoamericanas", Estudios Demográficos y Urbanos 21(3), pp. 625-661.
PRIETO, M. B. (2010) “Distribución espacial y segregación residencial de la población adulta mayor en el Gran Bahía Blanca, Argentina (1991 - 2001)", trabajo presentado en XVII Encontro Nacional de Estudos Populacionais, Caxambu, M. G., Brasil, 20 al 24 de septiembre de 2010.

REDONDO, N., DÍAZ FERNÁNDEZ, M., Llorente Marrón, M. M., Garay, S. Guidotti González, C., Mendoza Villavicencio, L. (2015). "El espacio residencial del cuidado de los adultos mayores en América Latina y España". Notas de Población, 100, pp. 223-258.

RODRÍGUEZ VIGNOLI, J. (2001) “Segregación residencial socioeconómica: ¿qué es?, ¿cómo se mide?, ¿qué está pasado?, ¿importa?" (LC/L.1576-P), Serie Población y Desarrollo 16, Santiago de Chile: Centro Latinoamericano y Caribeño de Demografía (CELADE).

SAAD, P. (2005) "Los adultos mayores en América Latina y el Caribe: arreglos residenciales y transferencias informales", Notas de Población 80, pp. 127-154.

STANFORD CENTER ON LONGEVITY (2015) The Spatial Distribution of an Aging Population, Stanford: Stanford University.

WHITE, M. (1983) “The Measurement of Spatial Segregation”, The American Journal of Sociology 88(5), pp. 1008-1018.

WINKLER, R., KLASS, R. (2012) “Residential segregation by age in the United States", Journal of Maps 8(4), pp. 374-378.

NOTAS

1 La ciudad de Córdoba, capital de la provincia homónima, se localiza a 703 kilómetros al Noroeste de la Ciudad Autónoma de Buenos Aires (ciudad capital de la República Argentina) y constituye el segundo aglomerado urbano del país, con una población de 1.329.604 habitantes, según los datos del último Censo Nacional de 2010. Demográficamente, la población de la ciudad puede ser caracterizada como envejecida, con poco más del $14 \%$ de las personas de 60 años y más.

2 Este término se utiliza para describir la posibilidad de cuidar, alimentar y guiar a las personas más jóvenes y contribuir a la generación siguiente (Hagestad y Uhlenberg, 2006).

3 Los valores de los indicadores de segregación calculados pueden verse afectados debido a la alta heterogeneidad en el tamaño y forma de los radios censales.

4 La expresión envejecer en la comunidad significa cubrir las necesidades asistenciales de la gente mayor frágil a fin de que puedan seguir viviendo en su propio hogar, o bien en una vivienda adecuada, de modo que se evite su ingreso en un centro especializado, es decir, su institucionalización. La opción de la gente mayor de envejecer en su entorno es, a priori, la mejor solución para respetar la dignidad, la voluntad y la independencia de la persona (Bosch Meda, 2006:88).
20 > Revista Márgenes № 19 Vol 13 > Diciembre 2016: 7 a 20 Facultad de Arquitectura > Universidad de Valparaíso
> ISSN electrónico 0719-4436
> ISSN impreso 0718-4034 